Sharp Laboratories Division, Beckman Instruments, Inc.

$\begin{array}{cccc}\text { Laboratory } & \begin{array}{c}\text { Published } \\ \text { number }\end{array} & \begin{array}{c}\text { Original date orence } \\ \text { rether value }\end{array} & \begin{array}{c}\text { Corrected date } \\ \text { or other value }\end{array} \\ \text { SL-3 } & 127: 109 & 239 \pm 52^{1} & \\ -8 & 127: 109 & 4020 \pm 100^{2} & \end{array}$

$\partial \mathrm{C}^{14}, \%$

Sequoia wood, tree-ring dated A.D. $1710 \pm 10$.

${ }^{2}$ C-1; A-219; LJ-175; TF-56.

\section{Socony Mobil Oil Corporation, Inc. ${ }^{1}$}

$\begin{array}{cccc}\begin{array}{c}\text { Laboratory } \\ \text { number }\end{array} & \begin{array}{c}\text { Published } \\ \text { reference }\end{array} & \begin{array}{c}\text { Original date or or value } \\ \text { other }\end{array} & \begin{array}{c}\text { Corrected date } \\ \text { or other value }\end{array} \\ \text { SM-1-8 } & 72: 111 & 22,400 \pm 1000 & \\ -13 & 72: 109 & 2300 \pm 200 \\ -14 & 72: 109 & 3150 \pm 200 \\ -15 & 72: 109 & 4110 \pm 250 \\ -16 & 72: 109 & 7650 \pm 250 \\ -17 & 72: 109 & 620 \pm 100 \\ -18 & 72: 109 & 2000 \pm 200 \\ -19 & 72: 110 & 3600 \pm 200 \\ -33 & 72: 111 & 22,800 \pm 1400 \\ -34 & 72: 111 & >30,0000 \\ -35 & 72: 111 & 22,600 \pm 1400 \\ -36 & 72: 111 & 10,270 \pm 450 \\ -38 & 72: 98 & 10,925 \pm 850 \\ -40 & 72: 98 & 11,840 \pm 800 \\ -43 & 72: 98 & 18,750 \pm 2000 \\ -44 & 72: 99 & 8265 \pm 650 \\ -46 & 72: 99 & 4300 \pm 1100 \\ -47 & 72: 99 & 24,400 \pm 4300 \\ -50 & 72: 111 & 11,400 \pm 600 \\ -50 \mathrm{C} & 72: 111 & 9900 \pm 500 \\ -57 & 72: 104 & 8080 \pm 600 \\ -58 & 72: 104 & 11,280 \pm 875 \\ -61 & 72: 104 & 4500 \pm 600 \\ -65 & 72: 105 & 12,520 \pm 1200 \\ -66 & 72: 105 & >25,000 \\ -68 & 72: 102 & 3540+3200 \\ & & \\ -69 & 72: 102 & 8575+4100 \\ -70 & 72: 101 & 2000 \pm 1000 \\ -72 & 72: 103 & 3925 \pm 800 \\ -73 & 72: 103 & 9800 \pm 750 \\ -74 & 72: 100 & 4450 \pm 200 \\ -75 & 72: 100 & 19,350 \pm 1200 \\ -76 & 72: 100 & 5623 \pm 200 \\ -77 & 72: 100 & 10,180 \pm 300 \\ -78 & 72: 101 & 2490 \pm 300 \\ -79 & 72: 101 & 3520 \pm 250 \\ -82 & 72: 102 & 2150 \pm 200 \\ -83 & 72: 102 & 5410 \pm 250 \\ -84 & 72: 103 & 3380 \pm 300 \\ -85 & 72: 103 & 10,320 \pm 700 \\ -86 & 72: 98 & 12,300 \pm 450 \\ -87 & 72: 99 & 20,750 \pm 1900 \\ -88 & 72: 103 & 2435 \pm 200 \\ -89 & 72: 103 & 9950 \pm 385 \\ -91 & 72: 98 & 4310 \pm 750 \\ -92 & 72: 98 & 12,360 \pm 2600 \\ & & \end{array}$

A.D./

B.C. date

A.D. 1711 2070 B.C.
A.D./

B.C. date

20,445 B.C.

345 B.C.

1195 B.C.

2155 B.C.

5695 B.C.

A.D. 1335

45 B.C.

1645 B.C.

20,845 B.C.

20,645 B.C.

8315 B.C.

8970 B.C.

9885 B.C.

16,795 B.C.

6310 B.C.

2345 B.C.

22,445 B.C.

9445 B.C.

7945 B.C.

6125 B.C.

9325 B.C.

2545 B.C.

10,565 B.C.

1585 B.C.

6620 B.C.

45 B.C.

1970 B.C.

7845 B.C.

2495 B.C.

17,395 B.C.

3668 B.C.

8225 B.C.

535 B.C.

1565 B.C.

195 B.C.

3455 B.C.

1425 B.C.

8365 B.C.

10,345 B.C.

18,795 B.C.

480 B.C.

7995 B.C.

2355 B.C.

10,405 B.C. 


\begin{tabular}{|c|c|c|c|c|}
\hline $\begin{array}{l}\text { Laboratory } \\
\text { number }\end{array}$ & $\begin{array}{l}\text { Published } \\
\text { reference }\end{array}$ & $\begin{array}{l}\text { Original date or } \\
\text { other value }\end{array}$ & $\begin{array}{l}\text { Corrected date } \\
\text { or other value }\end{array}$ & $\begin{array}{l}\text { A.D./ } \\
\text { B.C. date }\end{array}$ \\
\hline-96 & $72: 101$ & $10,500 \pm 1000$ & & 8545 B.C. \\
\hline-110 & $72: 103$ & $5170 \pm 900$ & & 3215 B.C. \\
\hline-111 & $72: 103$ & $8780 \pm 1300$ & & 6825 B.C. \\
\hline-115 & $72: 104$ & $>25,000$ & & \\
\hline-116 & $72: 98$ & $12,780 \pm 700$ & & 10,825 B.C. \\
\hline-117 & $72: 98$ & $12,460 \pm 700$ & & 10,505 B.C. \\
\hline-118 & $72: 100$ & $10,440 \pm 400$ & & 8485 B.C. \\
\hline-119 & $72: 100$ & $9340 \pm 400$ & & 7385 B.C. \\
\hline-121 & $72: 101$ & $2190 \pm 180$ & & 235 B.C. \\
\hline-122 & $72: 100$ & $3550 \pm 160$ & & 1595 B.C. \\
\hline-123 & $72: 100$ & $6910 \pm 300$ & & 4955 B.C. \\
\hline-124 & $72: 102$ & $5780 \pm 235$ & & 3825 B.C. \\
\hline-125 & $72: 101$ & $5935 \pm 300$ & & 3980 B.C. \\
\hline-128 & $72: 98$ & $7125 \pm 325$ & & 5170 B.C. \\
\hline .129 & $72: 99$ & $12,500 \pm 600$ & & 10,545 B.C. \\
\hline-130 & $72: 98$ & $5160 \pm 270$ & & 3205 B.C. \\
\hline-131 & $72: 98$ & $9610 \pm 500$ & & 7655 B.C. \\
\hline-132 & $72: 98$ & $8550 \pm 600$ & & 6600 B.C. \\
\hline .134 & $72: 105$ & $17,200 \pm 450$ & & 15,245 B.C. \\
\hline-135 & $72: 105$ & $18,500 \pm 500$ & & 16,545 B.C. \\
\hline-137 & $72: 105$ & $6070 \pm 170$ & & 4115 B.C. \\
\hline-138 & $72: 101$ & $2425 \pm 175$ & & 470 B.C. \\
\hline-139 & $72: 101$ & $3675 \pm 195$ & & 1720 B.C. \\
\hline-140 & $72: 104$ & $3615 \pm 190$ & & 1660 B.C. \\
\hline-141 & $72: 102$ & $5640 \pm 200$ & & 3685 B.C. \\
\hline .142 & $72: 103$ & $3800 \pm 150$ & & 1845 B.C. \\
\hline-143 & $72: 103$ & $7100 \pm 260$ & & 5145 B.C. \\
\hline-144 & $72: 104$ & $7260 \pm 260$ & & 5305 B.C. \\
\hline .145 & $72: 104$ & $3580 \pm 175$ & & 1625 B.C. \\
\hline-146 & $72: 104$ & $5850 \pm 400$ & & 3895 B.C. \\
\hline-147 & $72: 104$ & $17,050 \pm 600$ & & 15,095 B.C. \\
\hline .158 & $72: 99$ & $10,100 \pm 500$ & & 8145 B.C. \\
\hline-162 & $72: 105$ & $24,100 \pm 1300$ & & 22,145 B.C. \\
\hline-163 & $72: 105$ & $>30,000$ & & \\
\hline-169 & $72: 101$ & $5300 \pm 300$ & & 3345 B.C. \\
\hline$-169 \mathrm{C}$ & $72: 101$ & $5075 \pm 200$ & & 3120 B.C. \\
\hline-170 & $72: 102$ & $4250 \pm 175$ & & 2295 B.C. \\
\hline$-170 \mathrm{C}$ & $72: 102$ & $5260 \pm 260$ & & 3305 B.C. \\
\hline$-170 \mathrm{~F}$ & $72: 102$ & $7130 \pm 600$ & & 5175 B.C. \\
\hline-171 & $72: 102$ & $>30,000$ & & \\
\hline$-171 \mathrm{C}$ & $72: 102$ & $>30,000$ & & \\
\hline$-171 \mathrm{~F}$ & $72: 102$ & $>30,000$ & & \\
\hline-172 & $72: 99$ & $11,180 \pm 550$ & & 9225 B.C. \\
\hline$-172 \mathrm{C}$ & $72: 99$ & $11,380 \pm 400$ & & 9425 B.C. \\
\hline-173 & $72: 99$ & $22,750 \pm 1500$ & & 20,795 B.C. \\
\hline$-173 \mathrm{C}$ & $72: 99$ & $22,000 \pm 1000$ & & 20,045 B.C. \\
\hline-176 & $72: 105$ & $21,900 \pm 800$ & & 19,945 B.C. \\
\hline $.176 \mathrm{C}$ & $72: 105$ & $25,900 \pm 1850$ & & 23,945 B.C. \\
\hline-177 & $72: 105$ & $>30,000$ & & \\
\hline$-177 \mathrm{C}$ & $72: 105$ & $>30,000$ & & \\
\hline-339 & $72: 107$ & $550 \pm 220$ & & A.D. 1405 \\
\hline-340 & $72: 107$ & $1900 \pm 220$ & & A.D. 55 \\
\hline-341 & $72: 107$ & $4350 \pm 250$ & & 2395 B.C. \\
\hline-342 & $72: 107$ & $6200 \pm 300$ & & 4245 B.C. \\
\hline-344 & $72: 108$ & $4000 \pm 250$ & & 2045 B.C. \\
\hline-345 & $72: 108$ & $7700 \pm 600$ & & 5745 B.C. \\
\hline-346 & $72: 108$ & $8450 \pm 450$ & & 6495 B.C. \\
\hline-347 & $72: 108$ & $8950 \pm 350$ & & 6995 B.C. \\
\hline-348 & $72: 108$ & $1500 \pm 200$ & & A.D. 455 \\
\hline-349 & $72: 108$ & $3800 \pm 200$ & & 1845 B.C. \\
\hline-350 & $72: 108$ & $6150 \pm 200$ & & 4195 B.C. \\
\hline
\end{tabular}




$\begin{array}{cccc}\begin{array}{c}\text { Laboratory } \\ \text { number }\end{array} & \begin{array}{c}\text { Published } \\ \text { reference }\end{array} & \begin{array}{c}\text { Original date or } \\ \text { other value }\end{array} & \begin{array}{c}\text { Corrected date } \\ \text { or other value }\end{array}\end{array} \quad \partial \mathrm{C}^{14}, \%$

A.D./

B.C. date

A.D. 605

1445 B.C.

3295 B.C.

3345 B.C.

4445 B.C.

145 B.C.

3145 B.C.

6945 B.C.

7345 B.C.

7845 B.C.

4145 B.C.

4545 B.C.

1165 B.C.

2935 B.C.

5925 B.C.

10,225 B.C.

9995 B.C.

25,745 B.C.

${ }^{1}$ Reference standard: Hackberry wood grown about $1940,=94.6 \pm 1.2 \%$ NBS oxalic-acid standard.

\section{Salisbury, Rhodesia, University College, Department of Chemistry}

\begin{tabular}{|c|c|c|c|c|c|}
\hline $\begin{array}{l}\text { Laboratory } \\
\text { number }\end{array}$ & $\begin{array}{l}\text { Published } \\
\text { reference }\end{array}$ & $\begin{array}{l}\text { Original date or } \\
\text { other value }\end{array}$ & $\begin{array}{l}\text { Corrected date } \\
\text { or other value }\end{array}$ & $\partial \mathrm{C}^{14}, \%$ & $\begin{array}{c}\text { A.D./ } \\
\text { B.C. date }\end{array}$ \\
\hline SR-1 & $123: 32$ & $740 \pm 100$ & & & A.D. 1210 \\
\hline-2 & $123: 32$ & $1010 \pm 100$ & & & A.D. 940 \\
\hline$-\overline{3}$ & $123: 32$ & $560 \pm 100$ & & & A.D. 1390 \\
\hline-4 & $123: 32$ & $350 \pm 100$ & & & A.D. 1600 \\
\hline-5 & $123: 32$ & $750 \pm 100$ & & & A.D. 1200 \\
\hline-6 & $123: 36$ & $\Delta 260$ & $\Delta 260 \pm 10$ & $270 \pm 10$ & \\
\hline .7 & $123: 33$ & $>42,000$ & & & \\
\hline-3 & $123: 32$ & $560+100$ & & & 40.300 B.C. \\
\hline-9 & $123: 33$ & $42,200 \pm 2300$ & & & 40,300 B.C. \\
\hline-10 & $123: 33$ & $21,700 \pm 400$ & & & 19,750 B.C. \\
\hline-11 & $123: 33$ & $15,800 \pm 200$ & & & 13,850 B.C. \\
\hline-12 & $123: 33$ & $9400 \pm 100$ & & & 7450 B.C. \\
\hline-13 & $123: 33$ & $7690 \pm 140$ & & & 5740 B.C. \\
\hline-14 & $123: 34$ & $7610 \pm 110$ & & & 5660 B.C. \\
\hline-15 & $123: 34$ & $1220 \pm 100$ & & & A.D. 730 \\
\hline .17 & $123: 34$ & $1650 \pm 100$ & & & A.D. 300 \\
\hline-24 & $123: 35$ & $1930 \pm 100$ & & & A.D. 20 \\
\hline-25 & $123: 34$ & $650 \pm 120$ & & & A.D. 1300 \\
\hline-26 & $123: 35$ & $1420 \pm 120$ & & & A.D. 530 \\
\hline-28 & $123: 35$ & $800 \pm 100$ & & & A.D. 1150 \\
\hline-32 & $123: 34$ & $1100 \pm 100$ & & & A.D. 850 \\
\hline-33 & $123: 35$ & $1110 \pm 100$ & & & A.D. 810 \\
\hline-34 & $123: 35$ & $5200 \pm 140$ & & & 3250 B.C. \\
\hline-35 & $123: 34$ & $280 \pm 90$ & & & A.D. 1670 \\
\hline-36 & $123: 35$ & $720 \pm 120$ & & & A.D. 1230 \\
\hline-37 & $123: 35$ & $800 \pm 90$ & & & A.D. 1150 \\
\hline-38 & $123: 34$ & $1050 \pm 100$ & & & A.D. 900 \\
\hline-39 & $123: 33$ & $35,530 \pm 780$ & & & 33,580 B.C. \\
\hline
\end{tabular}

